\title{
Estudo do "Ages and Stages Questionnaires" com cuidadores de crianças institucionalizadas
}

\author{
Edson Junior Silva da Cruz - Universidade Federal do Pará, Belém, Brasil \\ Greicyani Brarymi Dias - Universidade Federal do Pará, Belém, Brasil \\ Janari da Silva Pedroso - Universidade Federal do Pará, Belém, Brasil
}

\begin{abstract}
Resumo
Este estudo explorou o conhecimento de cuidadoras sobre o desenvolvimento de crianças em acolhimento institucional com um instrumento de triagem. Participaram deste estudo quatro crianças na faixa etária de 5 anos de idade e as cuidadoras responsáveis. O instrumento utilizado foi o Ages and Stages Questionnaires, que contém 21 questionários que envolvem seis áreas de desenvolvimento. Os resultados revelaram que a comunicação foi uma das áreas pouco pontuadas pelas crianças. Suas principais dificuldades estão em verbalizar e se concentrar nas tarefas propostas. A área da coordenação motora ampla, que envolve, entre outras coisas, o correr e pular, incentivada pelo próprio ambiente da instituição, foi considerada dentro das expectativas para o desenvolvimento e recebeu pontuação máxima de acordo com o ASQ-3. As cuidadoras como pessoas de referência para as crianças, foram essenciais para aplicação do ASQ-3, que se mostrou sensível na identificação dos problemas do desenvolvimento. Palavras-chave: Criança institucionalizada; desenvolvimento infantil; Cuidadores; Questionário de triagem.
\end{abstract}

“Ages and Stages Questionnaires” study with caretakers of institutionalized children

\begin{abstract}
This study discussed the knowledge caretakers have on the development of children who live in institutional shelter, using a triage instrument. Took part of this study four five-year old children and the caretakers. The Ages and Stages Questionnaires were used, they are composed by 21 questionnaires which comprise six areas of development. The results demonstrated that communication was an area poorly scored by the children, their main difficulties are in verbalizing and focusing on the proposed task. The gross motor coordination field which gathers among other things jumping and running, all motivated by the environment of the institution, fit the expectations, hence received the maximum score according to ASQ- 3 . The caretakers, considered key people for the children, were essential for the application of ASQ-3, which had a major role in identifying developmental problems.

Keywords: Institutionalized children; Child development; Child caretakers; Triage questionnaire.
\end{abstract}

\section{Estudio del “Ages and Stages Questionnaires” con los cuidadores de los niños institucionalizados}

\begin{abstract}
Resumen
Este estudio ha explotado el conocimiento de cuidadoras sobre el desarrollo de niños en abrigo institucional con un instrumento de selección. Participaron de este estudio cuatro niños con edad mediana de 5 años de edad y las cuidadoras responsables. El instrumento utilizado fue el Ages and Stages Questionnaires que contiene 21 cuestionarios que abarcan seis áreas de desarrollo. Los resultados han revelado que la comunicación fue una de las áreas poco puntuadas por los niños, sus principales dificultades están en verbalizar y concentrarse en las tareas propuestas. El área de la coordinación motora amplia, que abarca entre otras cosas, el correr y saltar, está incentivada por el propio ambiente de la institución, fue considerada dentro de las expectativas para el desarrollo y ha recibido máxima puntuación de acuerdo con el ASQ-3. Las educadoras como personas de referencia para los niños fueron esenciales para la aplicación del ASQ-3 que se ha mostrado sencillo en la identificación de los problemas del desarrollo. Palabras clave: Niño institucionalizado; Desarrollo infantil; Educadores infantiles; Cuestionario de selección.
\end{abstract}

Este estudo, desenvolvido em uma instituição de acolhimento de crianças na faixa etária de 0 a 6 anos, localizada na cidade de Belém, investigou o conhecimento de cuidadoras a respeito do desenvolvimento de crianças por intermédio da escala de triagem - Ages and Stages Questionnaire Third Edition ASQ-3 (Squires, Bricker, Twombly \& Potter, 2009).

A qualidade dos cuidados primários é importante para o desenvolvimento da criança, sobretudo o contato íntimo e contínuo da mãe com o bebê. Nas instituições de acolhimento, as cuidadoras, como substitutas das mães, são as responsáveis pelo cuidado com as crianças, de modo que essas influenciam no desenvolvimento destas. É nessa relação primária que os especialistas julgam estar a base do desenvolvimento da personalidade e da saúde mental (Bowlby, 1995).

Estudos neurológicos que avaliam a influência de maus-tratos aos quais as crianças são expostas nos primeiros anos de vida apontam para problemas neuropsicológicos identificados a partir de uma avaliação cuidadosa (Dubowitz \& Poole, 2012; Margolin \& Vickerman, 2011; Walker, 2011; Wilson, Weaver, Cradock \& Kuebli, 2008). Tizard e Joseph (1970), ao analisarem as causas e variáveis neuropsicológicas do 
desenvolvimento de crianças, assinalam que os cuidados oferecidos nas instituições de acolhimento influem na manifestação de problemas relacionados ao desenvolvimento dessas, assim como na sua manutenção.

A esse respeito, Siqueira e Dell'Aglio (2010) apontam que o ambiente institucional não se constitui no melhor meio de desenvolvimento das crianças, pois o atendimento padronizado, o alto índice de criança por cuidador, a falta de atividades planejadas, a fragilidade das redes de apoio social e afetiva, além dos possíveis maus-tratos a que as crianças foram expostas antes de seu acolhimento, são fatores que influenciam no seu desenvolvimento e prejudicam a vivência institucional que opera no indivíduo. Os autores supracitados, em seus estudos com crianças cuidadas em instituições de acolhimento assinalam que as mesmas podem desenvolver problemas que afetem o seu pleno desenvolvimento, originando disfunções cognitivas, emocionais, perceptivas ou outras quaisquer. Segundo os autores esses problemas estão geralmente associados a fatores como: separação da família, condições físicas e sociais da instituição, onde a criança se encontra além do próprio cuidado destinado as mesmas.

Um dos meios de avaliar e prevenir o aumento desses problemas nas instituições de acolhimento, que prejudicam o desenvolvimento das crianças, pode ser empreendido a partir de uma avaliação permanente de seu desenvolvimento, mediante o uso de instrumentos padronizados. A utilização de escalas de avaliação permite detectar os problemas, as limitações presentes no desenvolvimento das crianças, como meio de prevenir dificuldades futuras e diminuir seus atrasos.

Há vários métodos de avaliação existentes destinados a identificar supostos atrasos no desenvolvimento infantil, como por exemplo, o da vigilância do desenvolvimento. Um dos instrumentos utilizado para avaliar o desenvolvimento de crianças é o ASQ3 , um questionário que pode ser aplicado por pais, cuidadores substitutos ou por profissionais da área que estão responsavéis pelas crianças. O ASQ-3 tem sido utilizado na triagem do desenvolvimento infantil nos mais variados contextos e gerado cada vez mais resultados confiáveis e importantes no sentido da valorização crescente dos responsáveis como avaliadores de suas crianças.

Schonhaut (2010) desenvolveu um estudo com 330 lactentes, seus respectivos pais e cuidadores, além de um profissional da área da saúde, responsável por aplicar a Escala de Avaliação do Desenvolvimento Psicomotor (EEDP) e compará-la com os resultados do
ASQ-3. O estudo apontou que o ASQ-3, por apresentar os resultados organizados em áreas, como comunicação, coordenação motora ampla e fina, resolução de problema e pessoal/social, constituiu-se como uma escala mais completa do que a EEDP, cujo diagnóstico na investigação do desenvolvimento psicomotor é baseado na pontuação total. Além disso, o autor reconheceu a importância de envolver os pais na avaliação dos filhos, a fim de contribuírem para as ações preventivas em saúde.

No Brasil, o ASQ-3 foi traduzido para o português e seus estudos de validação contaram com uma amostra de 18.942 crianças com idades entre 10 meses e 57 meses (Aguiar, 2011). Nos resultados, Aguiar observou que alguns itens precisariam ser adaptados à cultura brasileira, porém em relação à validação do ASQ-3 comprovou que é um bom instrumento para detecção de problemas no desenvolvimento infantil. Após a tradução, este instrumento passou a ser chamado pela equipe envolvida no estudo de ASQ-BR, sendo utilizado por professores e cuidadores de creches e pré-escolas do Rio de Janeiro.

Os resultados da aplicação do ASQ-3 nas creches da rede pública do Rio de Janeiro mostraram que 74\% das 46 mil crianças avaliadas tinham nível de desenvolvimento esperado para a sua idade. Revelou, ainda, que as crianças mais velhas apresentaram desempenho mais próximo das crianças norte-americanas, porém, a comparação entre os bebês indicou uma distância maior entre os dois universos (Avancine, 2012).

Os estudos supracitados e outros efetivados por ocasião da revisão da literatura desta pesquisa permitem afirmar que proceder às avaliações permanentes no desenvolvimento de crianças acolhidas em instituições é uma necessidade premente. Nesse contexto, importa abalizar a cautela na escolha de instrumentos para avaliar crianças, especialmente aquelas em situação de risco, além da necessidade de se obter informações de diferentes fontes sobre o desenvolvimento infantil. Justifica-se, portanto, a decisão do uso, nesta pesquisa, da escala ASQ-3, que, além de reconhecida por diversos profissionais e pesquisadores da área, é um instrumento de triagem adequado a ser aplicado com crianças em situação de acolhimento institucional.

\section{Método}

\section{Participantes}

O estudo envolveu quatro crianças em situação de acolhimento institucional, na faixa etária de cinco anos, 
pertencentes dos dormitórios VI e VII da instituição pesquisada, além de quatro cuidadoras responsáveis por essas crianças.

Como critérios de inclusão à aplicação da escala ASQ-3, utilizaram-se, além da idade das crianças (cinco anos), o tempo de permanência na instituição, superior a seis meses. Também se adotou como critério de inclusão selecionar as cuidadoras de referências das crianças. Outro critério adotado na seleção das cuidadoras diz respeito ao tempo de atuação na instituição, optando-se por aquelas que trabalhavam de três a cinco anos na mesma, com idade entre 26 a 34 anos.

\section{Local de pesquisa e especificidades}

A pesquisa foi realizada na cidade de Belém, em uma instituição destinada ao acolhimento de crianças na faixa etária de zero a seis anos, localizada na periferia, vinculada à Secretaria de Estado de Assistência e Desenvolvimento Social (SEDES), órgão do Governo do Estado do Pará.

A instituição possui espaço físico amplo, dividido em 20 ambientes, nos quais o trabalho é realizado. Abriga crianças com idades diferentes, isto é, em períodos de desenvolvimento distintos, e por esse motivo dispõe de espaços organizados e adaptados a esse atendimento diferenciado, pois buscam responder às demandas específicas das faixas etárias atendidas. $\mathrm{O}$ quadro funcional de cuidadoras, na ocasião da pesquisa, era composto por aproximadamente 60 funcionárias que dividiam a responsabilidade pelo atendimento às crianças acolhidas em tempo integral, pois a instituição funciona 24 horas.

\section{Instrumentos}

\section{Ages and Stages Questionnaires ASQ-3}

O ASQ-3 (Ages and Stages Questionnaires, $3^{\mathrm{a}} \mathrm{ed}$.), é composto de 21 questionários utilizados em crianças entre 1 e 60 meses. Possui 30 itens, organizados em 5 áreas que se relacionam com o desenvolvimento da comunicação, contendo itens referentes ao que se entende ou fala; à coordenação motora ampla, que avalia como a criança movimenta e usa seus braços e pernas enquanto joga ou brinca; à coordenação motora fina, relacionada ao movimento e coordenação das mãos e dedos da criança; à resolução de problemas, atinentes às habilidades para solucionar problemas e ao modo como as crianças brincam com brinquedos; além de itens relativos ao pessoal-social, que são as habilidades de autoajuda utilizadas pela criança em suas interações com outros.

Cada área do ASQ-3 abrange seis perguntas. Algumas destas compreendem ilustrações ao lado com o fito de facilitar o preenchimento do questionário. $\mathrm{O}$ instrumento abrange também uma seção para "observações gerais”, que contém informações adicionais acerca da criança, como auxílio para direcioná-la a um encaminhamento terapêutico adequado. Vale ressaltar que essa seção não será o foco principal deste estudo, porém foram considerados alguns aspectos para enriquecer a discussão sobre a triagem do desenvolvimento.

Para proceder à somatória referente à pontuação dos 30 itens que compõem o ASQ-3, adotam-se os seguintes critérios: quando o item marcado corresponde ao "sim", a pontuação atribuída é representada por 10 (dez) pontos, os quais indicam que a criança realizou o comportamento; quando o item marcado corresponde ao "às vezes", a pontuação corresponde a 5 (cinco) pontos, que indica uma resposta ocasional; e quando o item demarcado corresponde ao "ainda não", a pontuação vale 0 (zero), indicando que a criança ainda não realiza o comportamento avaliado. Os resultados devem ser analisados em uma folha específica para esse fim, chamada folha de compilação, a qual identifica a pontuação alcançada, que pode ser representada em uma tabela e/ou em gráfico, os quais retratam as classificações do desenvolvimento da criança investigada.

Após a soma por área das pontuações totais atingidas pela criança, deve-se comparar esse resultado com a pontuação limite da ASQ-3 e emitir o parecer final, que envolve o resultado da soma por área, os resultados das observações gerais e outros fatores adicionais, tais como se a criança tem a oportunidade para praticar as habilidades em que demonstrou algum risco. A soma dos pontos em cada uma das 5 áreas determinará em que classificação a criança se situa, representada pelos critérios: não necessita de avaliação; necessita de atividades adicionais com monitoração de sua evolução; requer avaliação aprofundada/direcionada feita por profissional.

\section{Procedimentos}

Autorização judicial para realização do estudo na Instituição

No intuito de realizar este estudo, solicitou-se preliminarmente uma autorização judicial para efetivação de visitas sistemáticas na instituição investigada, assim como para o acesso tanto aos arquivos 
documentais sobre a trajetória de vida e a condição sociofamiliar das crianças quanto à equipe de técnicos e cuidadoras que mantém contato diário com as crianças. Em concomitância à solicitação supracitada, o projeto também foi submetido ao Comitê de Ética em Pesquisa em Seres Humanos da Universidade Federal do Pará (CEP-ICS/UFPA), obtendo a devida autorização, mediante parecer.

Após o cumprimento das normas e exigências de pesquisas que envolvem seres humanos e as devidas autorizações, deu-se início à pesquisa de campo, por intermédio do acesso à instituição, tendo em vista o reconhecimento do ambiente institucional e dos sujeitos envolvidos no processo de investigação.

$\underline{\text { Reconhecimento do ambiente institucional e dos sujeitos }}$

A exploração do ambiente institucional se deu por intermédio de contatos iniciais com os participantes, mediante de abordagem individual para apresentar os objetivos e o método do estudo proposto. Buscou-se, com isso, motivar a participação das cuidadoras, assim como promover a receptividade da equipe técnica. Após esses contatos iniciais, deu-se início às orientações relacionadas à aplicação do questionário. Esse procedimento visou habilitar as cuidadoras no preenchimento dos questionários explicitados a seguir.

\section{Treinamento da equipe para aplicação da escala}

Os pesquisadores auxiliaram as cuidadoras no preenchimento dos questionários da escala ASQ-3. Os participantes foram treinados no manuseio da escala, mediante o preenchimento dos questionários, uma vez que mantinham contato permanente com as crianças, o que facilitou na obtenção de dados fidedignos representativos das crianças pesquisadas. Após essa etapa, a equipe partícipe da investigação procedeu à transcrição dos dados.

\section{Tratamento dos dados}

Após a coleta e a sistematização dos dados, os pesquisadores procederam a sua análise. $\mathrm{O}$ tratamento dos dados permitiu a identificação dos aspectos relacionados acerca da avaliação dos domínios, categorizados como: comunicação, coordenação motora ampla, coordenação motora fina, pessoal/social e resolução de problemas. As análises deram-se a partir da escala americana.

\section{Resultados e Discussão}

Para efeito de identificação dos partícipes da pesquisa na escala ASQ-3, definiu-se às quatro crianças a representação gráfica: C1, C2, C3 e C4. Em relação às cuidadoras que aplicaram as escalas, determinou-se a representação: E1, E2, E3 e E4.

Com a finalidade de determinar a classificação das crianças que se encontram no ASQ-3, seguiu-se a pontuação limite para cada área avaliada, presente no questionário. Um panorama das crianças é apresentado com as áreas do desenvolvimento acima dos limites determinados pelo instrumento ou que se mantinham próximas e abaixo dos limites, conforme apresentado na Tabela 1. Os resultados identificam se a criança está com o desenvolvimento normal, dentro das expectativas para sua idade, ou se requer encaminhamentos para avaliação mais aprofundada ou atividades adicionais.

$\mathrm{Na}$ categoria comunicação, as crianças C1, C3 e C4 obtiveram 40 pontos. Segundo a especificação da escala, essas crianças devem ser orientadas a fazer atividades adicionais para que obtenham melhor desempenho nessas atividades. A criança $\mathrm{C} 2$, em razão do ótimo desempenho nas atividades solicitadas pelo instrumento, alcançou 55 pontos, pontuação considerada como excelente pela ASQ-3.

$\mathrm{Na}$ categoria coordenação motora ampla todas as quatro crianças alcançaram um bom desempenho nas

Tabela 1. Resultados obtidos no ASQ-3

\begin{tabular}{lcccc}
\hline Categorias & C1-PL-PT/E & C2-PL-PT/ E & C3-PL-PT/E & C4-PL-PT/E \\
\hline Comunicação & $33.19-40 / A A$ & $33.19-55 / D N$ & $33.19-40 / A A$ & $33.19-40 / A A$ \\
Coordenação motora ampla & $31.28-60 / \mathrm{DN}$ & $31.28-60 / \mathrm{DN}$ & $31.28-60 / \mathrm{DN}$ & $31.28-60 / \mathrm{DN}$ \\
Coordenação motora fina & $26.54-25 / \mathrm{A}$ & $26.54-35 / \mathrm{AA}$ & $26.54-45 / \mathrm{DN}$ & $26.54-50 / \mathrm{DN}$ \\
Resolução de problema & $29.99-30 / \mathrm{AA}$ & $29.99-50 / \mathrm{DN}$ & $29.99-40 / \mathrm{AA}$ & $29-99-50 / \mathrm{DN}$ \\
Pessoal-social & $39.07-45 / \mathrm{AA}$ & $39.07-45 / \mathrm{AA}$ & $39.07-50 / \mathrm{DN}$ & $39-07-50 / \mathrm{DN}$ \\
\hline
\end{tabular}

Nota: PL=Pontuação limite; PT=Pontuação total; E=Encaminhamento; AA=Atividades adicionais; DN=Desenvolvimento normal; A=Avaliação. 
atividades realizadas, razão pela qual foram consideradas dentro do padrão de desenvolvimento normal, segundo a ASQ-3, obtendo a pontuação máxima pela escala. $\mathrm{Na}$ categoria coordenação motora fina, a criança C1 obteve um baixo desempenho nas atividades realizadas, motivo pelo qual, conforme orientação da escala, requer uma avaliação. A criança C2 obteve um desempenho melhor do que a $\mathrm{C} 1$, porém, segundo a escala necessita de atividades adicionais para melhorar sua coordenação motora fina. Já as crianças C3 e C4 tiveram seu desenvolvimento considerado normal, predominando uma boa avaliação da ASQ-3.

$\mathrm{Na}$ categoria resolução de problemas, as crianças C1 e C3 tiveram seus desempenhos avaliados dentro do limite de sua idade, assim foram orientadas a fazerem atividades adicionais. As crianças $\mathrm{C} 2$ e $\mathrm{C} 4$ tiveram seu desenvolvimento entendido como normal, obtendo uma boa avaliação, de acordo com a ASQ-3.

No que concerne à categoria pessoal-social, as crianças $\mathrm{C} 1$ e $\mathrm{C} 2$ foram orientadas a fazerem atividades adicionais, visto que seus desempenhos foram considerados dentro do limite de sua idade. Em relação às crianças C3 e C4, observou-se que realizaram bem as atividades que lhes foram propostas pela ASQ-3, portanto, seu desenvolvimento nesta categoria foi considerado normal para sua idade.

Segundo a classificação da ASQ-3, as quatro crianças avaliadas pelo estudo na instituição de acolhimento apresentaram as características identificadas a seguir. Em relação à C1, observou-se que seu desenvolvimento era normal apenas para coordenação motora ampla, saindo-se muito bem nas tarefas a ela encaminhadas, portanto mostrou boa habilidade nesta categoria.

Ruggiero e Suomi (2012), ao analisarem a categoria coordenação motora ampla, enfatizam que um dos motivos que pode influenciar o bom desenvolvimento da criança refere-se ao ambiente físico da instituição onde está inserida. Este é o caso específico da C1, que se encontra acolhida em uma instituição ampla, dotada de diversos ambientes propícios à sua estimulação, $\mathrm{o}$ que favorece a coordenação motora ampla.

$\mathrm{Na}$ categoria linguagem/comunicação a criança C1 atingiu um desenvolvimento dentro da zona limite. Das seis tarefas presentes no questionário, ela realizou com êxito a metade. Detectou-se que a criança compreendeu as ordens a ela dirigidas, porém demonstrou sérias dificuldades para verbalizar e se fazer entender, muitas vezes recorrendo a gestos para explicar o que queria dizer.
Em relação à categoria resolução de problema e pessoal/social, detectou-se que a C1 necessita de atividades adicionais e acompanhamento para se desenvolver normalmente, pois também se encontra no limite para atraso. Nessa categoria, a C1 apresentou um resultado preocupante nas tarefas solicitadas. Das seis tarefas a ela encaminhadas conseguiu realizar apenas três com sucesso e em outras três não obteve pontuação. No quesito pessoal/social que avalia especialmente as habilidades de autoajuda da criança e suas interações com outros, a $\mathrm{C} 1$ demonstrou como principal dificuldade compartilhar objetos com outras crianças.

A idade precoce de entrada na instituição e o tempo de acolhimento constituem-se como elementos centrais para discutir sobre os atrasos de desenvolvimento que podem afetar as crianças. Entretanto, em virtude do acolhimento precoce da maioria, antes mesmo do primeiro ano de idade, discute-se se as alterações são decorrentes dos maus-tratos sofridos antes do acolhimento ou se, ao contrário, são originadas na própria instituição, ou ainda, se esses locais funcionam como mantedores desses atrasos (Manson, García-Baamonde \& Alonso, 2011).

Essas situações certamente constituem-se como agravantes e prejudiciais, mormente considerando-se a situação do acolhimento. A vivência das mesmas pioram as condições dessas crianças e, sem a devida detecção e intervenção quando necessária, acirrar-se-ão os problemas em idades futuras, mediante o surgimento de outras incapacidades. Como exemplo, podem-se citar as dificuldades de crianças para algumas áreas essenciais da aprendizagem, caso este da C1, que tem sua coordenação motora fina comprometida, prejudicando-lhe a habilidade de escrever (Cardona, Facundo, Escobar, López, \& Ibáñez, 2012; Conti, Hansman, Heckman, Novak, Ruggiero \& Suomi, 2012; Schlesiger, Lorenz, Weinert, Schneider \& Roßbach, 2011).

A criança C2 apresentou um bom desempenho nas categorias comunicação, coordenação motora ampla e resolução de problemas. Na categoria comunicação, demonstrou entender e compreender as tarefas presentes no questionário, e no que concerne à expressão verbal, também não apresentou dificuldades. Em relação à coordenação motora ampla, seu desempenho foi avaliado como excelente, realizando as seis tarefas do quesito com sucesso. Na categoria resolução de problemas, a criança $\mathrm{C} 2$ demonstrou boa capacidade e desempenho dentro das expectativas para essa área (Conti e cols., 2012). 
Para as categorias coordenação motora fina e pessoal/social, a criança C2 demonstrou desempenho limítrofe, o que caracterizou o surgimento de atrasos nessa área, com indicação de encaminhamentos para atividades adicionais e monitoramento de seu desenvolvimento. $\mathrm{Na}$ categoria pessoal/social, a C2, apesar de demonstrar independência em certas habilidades da rotina da instituição, apresentou dificuldade em compartilhar objetos com outras crianças da instituição, assemelhando-se, neste aspecto, à C1.

Os indícios de atrasos para as áreas que se relacionam com as funções motoras, cognitivas e comportamentais, evidenciados neste estudo, são confirmados por autores que desenvolvem pesquisas acerca desta temática no mundo inteiro (Juffer, Palacios, Mary, Sonuga-Barker, Tieman, Bakermans-Kranenburg, Vorria \& Ijzendoorn, 2011). Dentre as áreas destacadas pelas escalas, as habilidades que fazem parte da coordenação motora, especialmente as que coordenam os grandes músculos, constituem-se como as mais mencionadas em estudos que avaliam o desenvolvimento de crianças em acolhimento institucional. $\mathrm{O}$ ato de mudar de posição, rastejar, engatinhar, sentar, firmar os pés no chão e andar são algumas aquisições que fazem parte dessa área.

Crianças pequenas necessitam explorar seu ambiente, interagir com pessoas e objetos de uma forma maior e melhor. Em função disso, carecem de cuidados mais direcionados e responsivos para que o desenvolvimento motor e demais áreas aconteça de uma forma natural e saudável (Bowlby, 1995).

Quando se fala em atrasos nas habilidades para a coordenação motora de crianças acolhidas em instituições infantis, questiona-se se as atividades de incentivo, como as de controle postural e mudança de posição, acontecem normalmente ou se essas crianças deveriam contar com um adulto para interagir e acompanhar as atividades e brincadeiras que estimulam o desenvolvimento motor. Esses e outros indícios de atrasos devem ser considerados após outros tipos de avaliações, como a história psicossocial da criança anterior à institucionalização.

As crianças $\mathrm{C} 3$ e C4 apresentaram resultados semelhantes em várias áreas abordadas pelo instrumento. Ambas tiveram excelentes resultados em praticamente todas as categorias. A criança C3 teve seu desenvolvimento considerado normal nos quesitos coordenação motora fina, coordenação motora grossa e na avaliação pessoal/social, realizando muito bem as atividades solicitadas. Nas demais categorias, comunicação e resolução de problemas, obteve um bom desempenho, porém, de acordo com o instrumento, precisa de atividades adicionais nesses dois quesitos (Dubowitz \& Poole, 2012).

A criança C3 demonstrou ter dificuldades de falar palavras que contêm a letra $\mathrm{L}$ e $\mathrm{R}$ e não soube nomear pelo menos quatro letras de seu nome, o que exige uma intervenção profissional a fim de melhorar sua comunicação. Também apresentou interesse sexual precoce, característica esta que pode ser justificada pela suspeita de abuso sexual do seu genitor, justificativa para a sua inserção na instituição de acolhimento.

Em relação à criança C4, identificou-se que obteve um excelente desempenho em quatro das cinco atividades que o instrumento aborda. Também apresentou um bom domínio de sua coordenação motora grossa e fina, realizando sem dificuldades as atividades solicitadas, tais como: segurar uma bola com as duas mãos quando outra pessoa joga; usando tesoura sem ponta a fim de cortar o papel ao meio, aproximadamente em linha reta; fazendo com que as lâminas se abram e se fechem.

A C4 também apresentou bons resultados nos itens resolução de problemas e pessoal/social; por exemplo, a criança conta até quinze sem errar, além de ir ao banheiro sozinha. A C4 demonstrou ter dificuldades em utilizar palavras que indicam comparação, como "mais pesado", "mais forte" ou "menor" e "maior", o que exige atividades adicionais, porém sem grandes intervenções, uma vez que sua comunicação é pouco comprometida, expressando-se bem quando deseja algo, utilizando-se corretamente do verbo no passado, além de responder às ordens solicitadas (Avancine, 2012).

Aspectos que se relacionam com a cognição e comunicação, avaliados pela escala ASQ-3 nas crianças que se encontram em acolhimento institucional, são muitas das vezes responsáveis pelos resultados de grande parte de dados indicativos para o atraso ou o limite do desenvolvimento. Alguns fatores que podem influir nos resultados encontrados são provenientes da história pregressa dessas crianças, presentes em seus prontuários.

As habilidades referentes à cognição e comunicação também são mediadas pelo nível de interação entre a criança e seu ambiente. O componente genético pode ter influência neste e em outros aspectos do desenvolvimento infantil, porém, sem a influência de um adulto emocionalmente estável, calmo e atencioso a promover e direcionar as interações da criança em seu meio, há dificuldades para se empreender o desenvolvimento dentro das expectativas almejadas, especialmente em 
áreas tão importantes para o aprendizado humano (Groeneveld, Vermeer, Van Ijzendoorn \& Linting, 2010; Manson, Garcia-Baamond \& Alonso, 2011).

A criança $\mathrm{C} 2$, presente há mais tempo na instituição do que as demais crianças, demonstrou um bom desenvolvimento dentro das expectativas. Isso pode estar relacionado com a hipótese de que, realmente, o espaço de acolhimento institucional funcionou favoravelmente no seu desenvolvimento, apesar das variáveis presentes em sua história psicossocial pregressa (quatro anos de acolhimento, genitora portadora de doença mental), que poderiam interferir negativamente na evolução saudável de seu desenvolvimento.

Quando se analisam as áreas presentes nos questionários ASQ-3 e o desempenho das quatro crianças, percebe-se que algumas habilidades deveriam receber intervenções precoces mais urgentes em razão de pontuarem negativamente, como, por exemplo, o caso das áreas da coordenação motora fina, pessoal/social e comunicação. A coordenação motora fina foi a área em que a criança $\mathrm{C} 1$ necessitou de mais auxílio e o agravante é que, possivelmente, isso só foi percebido após a avaliação da cuidadora. $\mathrm{Na}$ ocasião, a mesma mostrou-se surpresa com as dificuldades apresentadas pela criança, afirmando que relataria o caso para devidas providências pela equipe técnica.

Esses atrasos podem ser justificados pelas dificuldades dos profissionais em reconhecê-los, denotando problemas em perceber alterações no desenvolvimento considerado normal. Nesse sentido, cresce a preocupação com a qualificação dos profissionais que oferecem cuidados às crianças acolhidas, com o compromisso de lhes oferecer atenção individualizada, ainda que a proporção entre crianças e adultos seja em geral pouco favorável a essa prática.

Campos (2010) assinala que, em virtude à precarizada qualificação profissional, muitas crianças não são escutadas em suas necessidades, tampouco recebem estimulação adequada, principalmente por meio de situações lúdicas em interação com o educador. Isso pode estar relacionado ao fato desses profissionais não receberem instrução ou incentivos suficientes para realizar tais atividades.

$\mathrm{Na}$ área pessoal/social, as cuidadoras das quatro crianças referiram em seus relatos que uma das habilidades em que as crianças apresentam mais dificuldades refere-se ao compartilhamento dos objetos com seus pares. Isso se torna um problema nesse lugar, pois quase tudo é compartilhado entre as crianças. As cuidadoras analisam esse fato como algo negativo, pois essas crianças estão em um local que possui várias outras crianças; compartilhar certos objetos é comum para rotina e normas da instituição de acolhimento (Ruggiero \& Suomi, 2012).

Outra questão apontada foi a presença de agressividade cometida pela $\mathrm{C} 2$ em relação aos seus pares. A cuidadora responsável pela avaliação acredita que o tempo de acolhimento (4 anos) pode ser o grande responsável pela mudança de comportamento dessa criança no último ano. Além disso, a criança quase não tem atividades externas na instituição, o que a faz ficar o tempo todo nesse local. As cuidadoras que participaram deste estudo e avaliaram as crianças observaram que estas sentem falta de um ambiente familiar. Vale ressaltar que elas praticamente têm passado sua infância em ambientes de acolhimento institucional e que seu histórico é de pouco contato familiar, o que talvez justifique suas carências e agressividades diante de seus pares, na perspectiva do cuidador.

Longos períodos de institucionalização podem acarretar danos irreparáveis para o desenvolvimento infantil, principalmente quando ocorre durante os primeiros dois anos de idade (Bowlby, 1995). Oferecer cuidados de qualidade seria o mínimo que um local que se propõe a proteger dos maus-tratos poderia oferecer para quem ainda é dependente de todo estímulo para crescer e se desenvolver naturalmente. Um local que não oportuniza uma boa estrutura de vigilância em atenção precoce à criança pode contribuir, juntamente com outros fatores pré-natais, caso presentes, com o surgimento de adolescentes e adultos com sérios problemas de origem emocional/mental, que podem se traduzir, entre outras coisas, em comportamentos de agressividade difíceis de reverter (Hermenau, Hecker, Ruf, Schauer, Elbert \& Schauer 2011; Smyke, Koga, Johnson, Fox, Marshall, Nelson \& Zeanah, 2007).

A comunicação foi uma das áreas pouco pontuadas pela C1, C3 e C4; suas principais dificuldades estão em verbalizar e se concentrar nas tarefas propostas. As cuidadoras que avaliaram afirmaram que as crianças têm dificuldades em pronunciar algumas palavras, por exemplo, a criança $\mathrm{C} 1$ se comunica por meio de gestos, principalmente o apontar, quando quer algum brinquedo. Segundo a própria cuidadora, um dos motivos que pode justificar esse atraso é o fato de a C1 estar há muito tempo no espaço de acolhimento ( 3 anos), o que fez com que ela não tivesse os estímulos necessários para o desenvolvimento saudável para a comunicação.

Assim, como nas outras áreas, atrasos da comunicação não foram demonstrados igualmente por todas as 
crianças envolvidas no estudo, o que confirma os resultados de outras pesquisas em que a institucionalização parece não afetar da mesma forma as crianças nesses lugares (Smyke e cols., 2007). Agravos para o desenvolvimento infantil parecem persistir em idades posteriores e causar alguns transtornos, como, por exemplo, na vida escolar da criança, podendo a comunicação ser considerada como um desses agravos (Delcenserie, Genesee \& Gautheer, 2011).

Estudos apontam o acolhimento precoce por um longo período de tempo como um dos fatores que podem ocasionar alterações do desenvolvimento da linguagem. Tais eventos, muitas vezes, são identificados somente quando a criança inicia sua vida escolar (Campos, 2010). As intervenções são iniciadas para reverter os danos que deveriam ser tratados precocemente, porém, muitas vezes, as alterações e os danos permanecem, ainda que por um bom tempo, mesmo quando as crianças são colocadas em ambientes favoráveis para o seu desenvolvimento (Diamond e cols., 2003).

Um aspecto desta pesquisa alerta para algo há muito discutido, que é a qualidade dos cuidados dispensados às crianças, em que o elemento que interferirá para agravos no desenvolvimento dessa criança não será tanto o tempo de institucionalização em si, mas o tipo de atenção e interação que a criança recebe individualmente, o que esses autores chamam de "microcuidados", isto é, a forma como esses cuidados são dispensados a cada criança individualmente parece ser o grande modulador de o quanto esse desenvolvimento será favorecido ou não em idades posteriores.

\section{Considerações finais}

A pesquisa evidenciou a importância da avaliação de desenvolvimento infantil de crianças acolhidas institucionalmente, com o uso de um questionário de triagem que foi sensível para identificar problemas no desenvolvimento nas áreas da linguagem, coordenação motora ampla e coordenação motora fina, notando-se que as cuidadoras foram capazes de verificar atrasos desenvolvimentais das crianças por elas acompanhadas.

Reconheceu-se que a presença de uma cuidadora constante e de referência para a criança consegue avaliar de forma criteriosa, segundo o questionário de triagem. Portanto, instrumentalizar a equipe de cuidados para detecção de problema é um fator positivo no acompanhamento e cuidado de crianças acolhidas.

Neste estudo, o ASQ-3 foi capaz de identificar os atrasos na área da linguagem das crianças na instituição.
Tal identificação confirma, com os estudos apresentados na discussão, que os problemas na linguagem são indicadores negativos do processo de acolhimento institucional, principalmente quando considerada a variável tempo. Ainda, notou-se que o ambiente físico da instituição influenciou positivamente no desempenho motor amplo avaliado no questionário.

Reconheceu-se que a quantidade de crianças avaliadas nesta pesquisa foi reduzida, o que impossibilita generalizações analíticas sobre o desenvolvimento de crianças em acolhimento institucional. Em relação à aplicação do instrumento, a baixa escolaridade do educador pode dificultar o entendimento das questões avaliadas.

Sugerem-se outros estudos de avaliação de desenvolvimento infantil com instrumentos psicológicos validados no Brasil e para a realização de uma análise comparativa do ASQ-3. Desse modo, reforçaria a importância da avaliação realizada por cuidadoras na detecção de problemas no desenvolvimento.

\section{Referências}

Aguiar, A. M. A. (2011). Avaliação do desenvolvimento, trajetórias de socialização e o ASQ 3. Anais do $7^{\circ}$ congresso Norte e Nordeste de psicologia, Bahia. Anais. Disponível em: http:// www.conpsi7.ufba.br.

Avancine, M. (2012). Revista Educação, edição 179 - março 2012 - reportagem in seção Políticas Públicas; medida para infância. São Paulo: Segmento. Disponível em: http: \\www.editorasegmento. com.br.

Bowlby, J. (1995). Cuidados maternos e saúde mental. $3^{\mathrm{a}}$ ed. São Paulo: Martins Fontes.

Campos, A. R. S. (2010). O momento da avaliação na intervenção precoce: o envolvimento da família estudo das qualidades psicométricas do ASQ-2 dos 30 aos 60 meses. Revista Brasileira de Educação Especial, 16(2), 177-196. Doi:10.1590/ S1413-65382010000200003.

Cardona, J. F., Manes, F., Escobar, J., \& López, J. (2012). Potentials consequences of abandonment in prescholl-age: neuropsychologial findings in institucionalized children. Behavioral Neurology, 25, 1-11. Disponível em: http: /iospress.metapress.com/ contente/Doi:b459ow27581g21822.

Conti, G., Hansman, C., Heckman, J. J., Novak, M. F. X., Ruggiero, A., \& Suomi, S. J. (2012). Primate

Psico-USF, Bragança Paulista, v. 19, n. 3, p. 411-420, set./ dez. 2014 
evidence on the late health effects of early life adversity. Procledings of the national academy of the national academy of sciences of the United States of America, 109(3), 8866-8871.

Delcenserie, A., Genese, F., \& Gautheer, K. (2011). Language abilities of internationally adapted children from China during the early school years: Evidence for early age effects? Applied psycholinguistics, 01-28.

Diamond, G. W., Senecky, Y., Schurr, D., Zuckerman, J., Inbar, D., Eidelman, A., \& Cohen, H. J. (2003). Pre-placement screening in international adoption. Israel Medical Association Journal. 5, 763-766. Disponível em: http://www.ima.org.il/imaj/ar3nov-1. pdf.

Dubowitz, H., \& Poole, G. (2012). Em: H, Tremblay, R. E. Macmillan, M. Boivin, R. D. Peters. (Eds), Encyclopedia on early childhood development and strategic knowledge cluster on early Child development. Montreal, Quebec: Centre of excelence for early Child hood development and strategic knowledge cluster on early Child development. Child neglect: $\mathrm{Na}$ overview. pp.01-06. Disponível em <http://www.child-encyclopedia.com/pages / pdf/ Dubowitz-poolANGXP1.pdf.

Groeneveld, M. G., Vermeer, H. J., Ijzendoorn, M. H. V., \& Linting, M. (2010). Stress, cortisol and well of caregivers and children in home-based child care: a case for differential susceptibility. Child: Care, Health and Development, 38(2), 561-560. Disponível em: http://onlinelibrary.wiley.com/ Doi:10.1111/j.1365-2214.2010.01194.x/pdf.

Hermenau, K., Hecker, T., Ruf, M., Schauer, E., Elbert, T., \& Schauer, M. (2011). Childhood adversity, mental ill-health and aggressive behavior in an African orphanage: changes in response to traumafocused therapy and the implementation of a new instructional system. Child Adolesc Psychiatry Ment Health, 5(29). Doi: 10.1186/1753-2000-5-29PMCI D:PMC3189861.

Juffer, F., Palacios. J., Mary, L. L., Sonuga-Barker, E. J. S., Tieman. W., Bakermans-Kranenburg, M. J., Vorria, P., \& Ijzendoorn. M. H. V. (2011). II Development of adopted children with histories of early adversity. Monographs of the Society for Research in Child Development, 76(4), 31-61. Doi:10.1111/j.1540-5834.2011.00627.x/pdf.
Manson, J. M. M., Garcia-Baamond, M. E., \& Alonso, M. B. (2011). Design of a language stimulation program for children suffering abuse. Children and Youth Services Review, 33(7), 1325-1333. Doi: 10.1016/j.childyouth.2011.03.0031.

Margolin, G., \& Vickerman, K. A. (2011). Posttraumatic stress in children and adolescents exposed to family violence: I. Overview and issues. Cougle and family. Psychology: Research and Practice, 1, 63-73. Doi:10.1037/2160-4096.1.563.

Schlesiger, C. (2011). From birth to early Child care. Zlitschriftn fur erziehungs wissenschaft. 14 (2), 187202. Disponível em: http://springerlink.com/ content/174n0w56rq741627//.

Schonhaut, L. (2012). Comparacion de La evoluciòn tradicional del desarrollo psicomotor versus uma pruela auto administrada. Rev. Chil. Pediatr., 87(6), 498-505. Disponível em: http://www.scielo.cl/ scielo.php?pid=Doi:50370-41062010000600003.

Siqueira, A. C., \& Dell'Aglio, D. D. (2010). Crianças e adolescentes institucionalizados: desempenho escolar, satisfação de vida e rede de apoio social. Psicologia: Teoria e Pesquisa, 26(3), 407-475. Disponível em http://www.scelo.br/pdf/ptp/v2n3/ a03v26n3.

Smyke, A. T., Koga, S. F., Johnson, D. E., Fox, N. A., Marshall, P. J., Nelson, C. A., \& Zeanah, C. H. (2007). The caregiving context in institution-reared and family-reared infants and toddlers in Romania. Journal of Child Psychology and Psychiatry, 48(2), 210-218. Disponível em: http://www.ncbi.nlm. nih.gov/pubmed.

Squires, J., Bricker, D., Twonby, E., \& Potter, L. (2009). Ages e Stages Questionnaires, third edition (ASQ-3): User's guide. San Antonio, TX: Paul H. Brookes Publishing.

Tizard, B., \& Joseph, A. (1970). Cognitive development of Young children in semidential care: a study of children aged 24 months. Jornal of Child Psychology and Psychiatry, 11(3), 177-186. Doi: 10.1111/j.14697610.1970.tb01024.x/pdf.

Walker, P. S. (2011). Inequality in early childhood: risk and protective fator for early. Child Development, 378(9799), 1325-1338. Doi: 10.1016/50140-6736(11)60555-2. 
Wilson, S. L., Weaver, T., Cradock, M., \& Kuebli, J. (2008). Apriliminary study of the cognitive and motor skills acquisitions of Young international adoptees. Children and Youth Services Review, 30(5), 585-596.
Recebido em: $13 / 11 / 13$

Reformulado em: 02/03/2014

Segunda reformulação em: 13/04/2014

Aprovado em: 19/05/2014

Sobre os autores:

Edson Júnior Silva da Cruz é assistente social, mestrando em Psicologia pela Universidade Federal do Pará - PPGP/ UFPA, membro do Laboratório de Desenvolvimento e Saúde - LADS.

E-mail: edsoncruzufpa@gmail.com

Greicyani Brarymi Dias é terapeuta ocupacional, mestra em Psicologia pela Universidade Federal do Pará - PPGP/ UFPA e membro do grupo: Pesquisa e Ações em Psiquiatria e Psicologia da Saúde - UFPA.

E-mail: greicypimenta@yahoo.com.br

Janari da Silva Pedroso é psicólogo, doutor em Ciências Sócio-Ambientais (Universidade Federal do Pará/NAEA), pós-doutorando em Psicologia (Universidade Católica de Brasília), professor associado 1 da Faculdade de Psicologia e do Programa de Pós-Graduação em Psicologia da Universidade Federal do Pará - PPGP/FAPSI/UFPA. Coordena o Laboratório de Desenvolvimento e Saúde - LADS. Pesquisador CNPq.

E-mail: pedrosoufpa@gmail.com

Contato com os autores:

Edson Júnior Silva da Cruz

Rua Antônio Pontes, 1373. Centro.

CEP: 68790-000. Cidade: Santa Isabel do Pará - Pará. 